\title{
Smad1/5 signal transduction regulates the ameloblastic differentiation of induced pluripotent stem cells
}

\section{Zhi LIU(a) iD \\ Guang ZENG(b) iD \\ Qing QIN(c) iD \\ Cong SUN(a) iD \\ Lei $\operatorname{TAN}^{(a)}$ iD \\ (a) Xi'an Jiaotong University, The First Affiliated Hospital, Department of Oral Sciences, Xi'an, China; \\ (b)Forth Military Medical University, Tangdu Hospital, Department of Dentistry, Xi'an, China; \\ (c)The 323rd Hospital of PLA ${ }^{3}$ Department of Dentistry, Xi'an, China.}

Declaration of Interests: The authors certify that they have no commercial or associative interest that represents a conflict of interest in connection with the manuscript.

\section{Corresponding Author:}

Zhi Liu

E-mail: 70545084@qq.com

hitps://doi.org/10.1590/1807-3107bor-2020.vol34.0006

Submitted: July 13, 2018

Accepted for publication: November 27, 2018

Last revision: January 28, 2019
Abstract: Induced pluripotent stem (iPS) cells could be induced into ameloblast-like cells by ameloblasts serum-free conditioned medium (ASF-CM), and bone morphogenetic proteins (BMPs) might be essential during the regulation of this process. The present study investigates the signal transduction that regulates the ameloblastic differentiation of iPS cells induced by ASF-CM. Mouse iPS cells were characterized and then cultured for 14 days in epithelial cell medium (control) or ASF-CM. Bone morphogenetic protein receptor II (BMPR-II) siRNA, inhibitor of Smad1/5 phosphorylation activated by activin receptor-like kinase (ALK) receptors, and inhibitors of mitogen-activated protein kinases (MAPKs) phosphorylation were used to treat the iPS cells in combination with ASF-CM. Real-time PCR, western blotting, and immunofluorescent staining were used to evaluate the expressions of ameloblast markers ameloblastin, enamelin, and cytokeratin-14. BMPR-II gene and protein levels increased markedly in ASF-CMtreated iPS cells compared with the controls, while the mRNA levels of Bmpr-Ia and Bmpr-Ib were similar between the ASF-CM and control groups. ASF-CM stimulation significantly increased the gene and protein expression of ameloblastin, enamelin and cytokeratin-14, and phosphorylated SMAD1/5, p38 MAPK, and ERK1/2 MAPK compared with the controls. Knockdown of BMPR-II and inhibition of Smad1/5 phosphorylation both could significantly reverse the increased expression of ameloblastin, enamelin, and cytokeratin-14 induced by ASF-CM, while neither inhibition of p38 nor ERK1/2 phosphorylation had significant reversing effects. We conclude that smad1/5 signaling transduction, activated by ALK receptors, regulates the ameloblastic differentiation of iPS cells induced by ameloblast-conditioned medium.

Keywords: Induced Pluripotent Stem Cells; Bone Morphogenetic Protein Receptors, Type II.

\section{Introduction}

Stem-cell-based tissue-engineering approaches are being considered as a potential method to generate tooth tissues that closely match the physical and biomechanical properties of natural teeth. ${ }^{1}$ Many studies have reported that dental stem cells, such as dental pulp stem cells (DPSCs), periodontal ligament stem cells (PDLSCs), stem cells from exfoliated 
deciduous teeth (SHEDs), or from the apical papilla (SCAPs), are multipotent and can form structures resembling tooth tissue. These dental stem cells have been applied successfully to generate dentin pulp and the periodontal ligament cement complexes. ${ }^{2,3}$ However, the shortage of a patient's own dental stem cells limits the use of this approach.

Induced pluripotent stem (iPS) cells are considered as a new cell source for tooth regeneration, resulting from the rapid progress of iPS cells generation and the related technical platforms. ${ }^{4}$ Arakaki et al. ${ }^{5}$ developed a dental epithelial cell line from the cervical loop at the apical end of the lower incisors, and demonstrated that co-culturing with this epithelial cell line could induce iPS cells to adopt an ameloblastlike phenotype, such as displaying an epithelial cell-like morphology with positive expressions of the ameloblast markers ameloblastin, enamelin (ENAM), and cytokeratin-14 (CK14). However, Yoshida et al. ${ }^{6}$ demonstrated that mouse iPS cells could be induced into ameloblast-like cells more efficiently by culturing them with the conditioned medium of epithelial cell rests of Malassez (ERM) cells compared with co-culturing with ERM cells. Furthermore, Liu et al. showed recently that ameloblast serum-free conditioned medium (ASF-CM) could induce ameloblast-like cells from mouse iPS cells successfully, which was evident by the positive expression of ameloblast markers AMBN and CK14. They further found that culturing mouse iPS cells in ASF-CM supplemented with bone morphogenetic protein 4 (BMP4) promoted odontogenic differentiation. By contrast, culturing mouse iPS cells in ASF-CM supplemented with the BMP inhibitor noggin reversed the above effect. ${ }^{7}$ These results highlighted the promising future of tooth regeneration from iPS cells and indicated that growth factors from BMPs family could be essential during the regulation of this process. Therefore, the regulatory mechanism of the ameloblastic differentiation of iPS cells should be investigated further to promote the application of iPS in tooth regeneration.

BMPs, members of the transforming growth factor $\beta$ (TGF- $\beta$ ) superfamily, are multi-functional cytokines that regulate the growth and differentiation of many cell types, and play a major role in early embryogenesis and in subsequent organogenesis. BMP signaling is mediated via the activation of combinations of type I and type II serine/threonine kinase receptors. These receptors' signals have been reported to activate the canonical TGF- $\beta$ signaling proteins termed SMAD proteins. ${ }^{8}$ However, recent studies have also confirmed that BMPs might also activate the mitogen-activated protein kinase (MAPK) pathways directly, including P38, ERK1/2, and JNK. ${ }^{9}$ In addition, previous studies have shown that BMPs are important regulators in dental morphogenesis, including the epithelialmesenchymal interaction and epithelial bud development. ${ }^{10}$ These findings have generated much interest in the effects of BMPs on the differentiation of mouse iPS cells into the odontogenic lineage. However, the signal transduction pathways that are active during the ameloblastic differentiation of iPS cells remain unknown.

In the present study, we explored the mechanism of the ameloblastic differentiation of iPS cells induced by ameloblast-conditioned medium. A BMP receptor 2 (BMPR-2) short interfering RNA (siRNA) was used to investigate the involvement of BMPR-2 in this process. Furthermore, the involvement of SMAD1/5 and MAPKs signaling in the ameloblastic differentiation of iPS cells was also evaluated.

\section{Methodology}

\section{Culture and characterization of mouse iPS cells}

The mouse iPS cells (C5 cell line) were obtained from the South China Institute for Stem Cell Biology and Regenerative Medicine, Chinese Academy of Sciences. The iPS cells were cultured as previously described. ${ }^{7}$ Briefly, mouse embryo fibroblasts treated with mitomycin- $C$ were used as the feeder cells for iPS cells, and Dulbecco's modified Eagle's medium (Gibco, Paisley, UK) supplemented with fetal bovine serum (Gibco), penicillin-streptomycin, $\beta$-mercaptoethanol (Sigma-Aldrich, St. Louis, USA), sodium pyruvate (Gibco), and leukemia inhibitory factor (Chemicon, Billerica, USA) was used as the culture medium. To 
characterize the mouse iPS cells $(n=6)$, the protein expression of the embryonic stem cell (ESC)-specific markers OCT4 and SSEA-4 and the differentiated ESC marker SSEA-1 were detected by immunofluorescent staining. All experimental procedures were performed according to institutional guidelines previously approved by the Ethics Committee of the First Affiliated Hospital of Xi'an Jiaotong University.

\section{Preparation of ameloblast serum-free conditioned medium (ASF-CM)}

ASF-CM was prepared as previously reported. ${ }^{7}$ Briefly, the mandibular incisors isolated from forty 7-day-old mice were used to isolate the ameloblasts (Supplementary Figure 1), and all mesenchymal cells were removed by repeated purification using trypsin (Gibco, Paisley, UK). Epithelial cell medium (Sciencell, Carlsbad, USA) was used to culture the purified cells, and was changed every $2 \mathrm{~d}$. The supernatant was then collected, filtered, and stored at $-80^{\circ} \mathrm{C}$. In order to assure the reproducibility of the experiment, we have mixed all the supernatants together, and then used them as ASF-CM for the subsequent experiments. In this way, the active components in the conditioned media were the same among the experiments.

\section{RNA interference}

The mouse BMPR-II siRNA sequence was synthesized by Invitrogen (Invitrogen, Life Technologies, Grand Island, USA), and the siRNAs were transfected into cells as previously described. ${ }^{11}$ Briefly, the nucleotide target sequence of BMPR-II was GCA GTA CTA GTT CTA GCT TGC, and the sequence directed to the non-expressed mRNAs (ACG UGA CAC GUU CGG AGA ATT) was used as a control. 1 $\times 10^{6}$ iPS cells were seeded into each well of six-well plates. After $24 \mathrm{~h}$ of incubation, lipofectamine 2000 (Invitrogen, USA) was used to transfect the BMPR-II siRNAs or the controls into the iPS cells, following the manufacturer's protocols $(n=6)$. Twenty-four hours after transfection, the protein level of BMPR-II were determined by western blotting.

\section{In vitro differentiation of mouse iPS cells}

In the control group, the iPS cells were cultured in epithelial cell medium, which was used to create the ASF-CM. For the Bmpr-II knockdown experiment, the iPS cells treated with BMPR-II siRNA or the control vector were cultured separately in ASF-CM or epithelial cell medium (Control) for $14 \mathrm{~d}(\mathrm{n}=6)$. For the pathway inhibition experiment $(n=6)$, the iPS cells were cultured in epithelial cell medium (Control), ASF-CM, or ASF-CM supplemented separately with 200 nM LDN-193189 (a selective SMAD1/5 inhibitor activated by activin receptor-like kinase (ALK) receptors; Selleck, Houston, USA), 10 M SB203580 (a selective p38 inhibitor; Cell Signaling Technology, Danvers, USA), or $10 \mu \mathrm{M}$ U0126 (a selective ERK1/2 inhibitor; Cell Signaling Technology). All the control and conditioned media were changed every $2 \mathrm{~d}$ during the 14-day experimental period.

\section{Immunofluorescent staining}

The iPS cells were fixed by $4 \%$ paraformaldehyde, and permeabilized by $0.1 \%$ Triton X-100 (Sigma-Aldrich, St. Louis, USA). ${ }^{12}$ To characterized the iPS cells, the specimens $(n=6)$ were incubated overnight at $4^{\circ} \mathrm{C}$ with mouse anti-OCT4 (1:100), mouse anti-SSEA-4 (1:100) or mouse anti-SSEA-1 (1:100), and were then incubated with a Cy3-conjugated secondary antibody (1:100, Molecular Probes, Breda, Netherlands) for 1 $\mathrm{h}$, and subsequently with 4',6-diamidino-2-phenyl indole (DAPI) for $3 \mathrm{~min}$ at room temperature. To evaluate the ameloblastic differentiation of iPS cells $(\mathrm{n}=6)$, goat anti-ameloblastin (1:100), goat antienamelin (1:100), rabbit anti-CK14 (1:100) primary antibodies, and FITC-labeled secondary antibody (1:200; Chemicon, Billerica, USA) were used. All primary antibodies were purchased from the Santa Cruz Biotechnology (Dallas, USA). Samples ( $\mathrm{n}=$ 6) were examined under a confocal microscope (FV1000, Olympus, Japan). Five fields of view at $400 \times$ magnification were selected at random, and the percentage of the number of immune-positive cells to total cells in each field was counted and averaged for statistical analysis.

\section{Reverse transcription PCR (RT-PCR)}

Gene expressions of Ambn, ENAM, Ck14, dentine matrix protein-1 (Dmp-1), and dentine sialoprotein $(D s p)$ were detected as described previously. ${ }^{12}$ Briefly, total RNA from the iPS cells cultured under each 
differentiation condition was extracted using Trizol (Thermo Fisher Scientific, Grand Island, USA). A 7500 real-time PCR machine (Thermo, USA) was used to analyze the expression of the target genes using glyceraldehyde 3-phosphate dehydrogenase (Gapdh) as the internal control. The results were calculated as the relative quantification compared to the control group. Primer sequences for the genes are shown in Table. Data were collected from six independent pooled samples $(n=6)$.

\section{Western blotting}

Total protein from the iPS cells $(40 \mathrm{mg})$ was fractionated by sodium dodecyl sulfatepolyacrylamide gel electrophoresis (Bio-Rad, Martinez, USA) and transferred onto a nitrocellulose membrane. The membrane was blocked with $5 \%$ non-fat milk and incubated with primary antibodies against phospho-SMAD1/5 (1:800, 9516), glyceraldehyde-3-phosphate dehydrogenase (GAPDH, 1:1000, 2118), phospho-p38 (Thr180/ Tyr182) (1:800, 451), total-p38 (1:1000, 9212), phosphoERK1/2 (Thr202/Tyr204) (1:800, 4370), total-ERK1/2 (1:1000, 4695), phospho-JNK (1:800, 4688), and totalJNK $(1: 1000,9252)$. All antibodies were obtained from Cell Signaling Technology (Cell Signaling Technology, Danvers, USA). Signals were visualized by incubation with a horseradish peroxidaseconjugated secondary antibody (1:5000, Zhongshan Golden bridge Biotechnology, China) and enhanced chemiluminescence detection. ${ }^{12}$ The stained bands were scanned and quantified using a densitometer (Syngene Bioimaging System; Frederick, USA) and Scion Image software (Frederick). Protein expression levels were normalized against $\beta$-actin for each sample. Data were collected from three independent pooled samples $(n=3)$.

\section{Statistical analyses}

The measurement of cell number was performed blindly by two independent observers (ZG and QQ) using Photoshop CS 12.0 software (Adobe, USA) and there was a high level of agreement between the two observers (all $r>0.9$ ), thus the mean of the two measurements was used for statistical analysis. All data were reported as mean \pm standard deviation (SD) for each group. The Shapiro-Wilk test was used to assess the normality of the distribution of the data and Levene's test was used to assess the homogeneity of variance, and the assumptions of parametric tests were fulfilled for all data. ${ }^{12}$ Therefore, the statistical significance among groups was evaluated by analysis of variance (ANOVA) with post hoc comparison between groups by Tukey's test. P-values less than 0.05 were considered statistically significant in all cases.

\section{Results}

\section{Ameloblast-conditioned medium induced ameloblastic differentiation of iPS via BMPR-II}

The iPS colonies were positive for embryonic stem cell (ESC)-specific markers OCT4 and SSEA-4, but were almost completely negative for differentiated ESC marker SSEA-1 (Figure 1A), indicating the ESClike properties. To clarify the mechanism underlying the ameloblastic differentiation of iPS induced by ASF-CM, we cultured the iPS cells for 14 days in the control medium or ASF-CM, and the gene

Table. Primers for quantitative real-time RT-PCR.

\begin{tabular}{lcc}
\hline Genes & Sequence (Forward) & Sequence (Reverse) \\
\hline AMBN & CCTTGAGACAATGAGACAGTTGGG & CCTGGGTAAAAAGCGGATGC \\
ENAM & TCACACCCTTCAGCCTCATCA & CCACTTCGGTTCTCTCATTTTCTG \\
CK14 & CCCTACTTCAAGACCATTGAGGAC & TCATGCGCAGGTTCAACTCT \\
Dmp-1 & TCAGGACAGTAGCCGATCCAAAG & ATGGGTTTGTTGTAAGCATCA \\
Dsp & CTCGGAGGCTTTGAAGACATTGA & GCTGCAGTTCCTGGATGTGTTAGA \\
GAPDH & CACCATGGAGAAGGCCGGGG & GACGGACACATTGGGGGTAG \\
\hline
\end{tabular}


expression levels of different BMP receptors were analyzed. As shown in Figure 1B, Bmpr-II mRNA levels were markedly increased in ASF-CM-treated iPS cells compared with the controls $(p=0.013)$, while the mRNA levels of Bmpr-Ia and Bmpr-Ib were similar between the ASF-CM and control group $(p>0.05)$. A similar increase in BMPR-II protein levels was observed by western blotting analysis $(p=0.022$, Figure $1 C)$, indicating that ASF-CM could induce the expression of BMPR-II. To further confirm the involvement of BMPR-II in the ameloblastic differentiation of iPS cells, we pre-treated the iPS cells with a BMPR-II siRNA before culturing them in control medium or ASF-CM, and then detected the expression of ameloblast-specific genes (Ambn, ENAM and Ck14) and odontoblast-specific (Dmp-1 and Dsp) genes. The transfection efficiency of BMPR-II siRNA is about $82 \%$, and the protein level of BMPR-II was reduced dramatically by the BMPR-II siRNA $(p<0.05$, Figure 1C). The PCR results showed that ASF-CM stimulation significantly increased the gene expression of Ambn, ENAM, and Ck14 compared with that of the control, while BMPR-II siRNA obviously attenuated the above ASF-CM-induced effects (all $\mathrm{p}<0.05$, Figure 2). However, the gene expression of Dmp-1 and Dsp was similar among the control, ASF-CM,

A
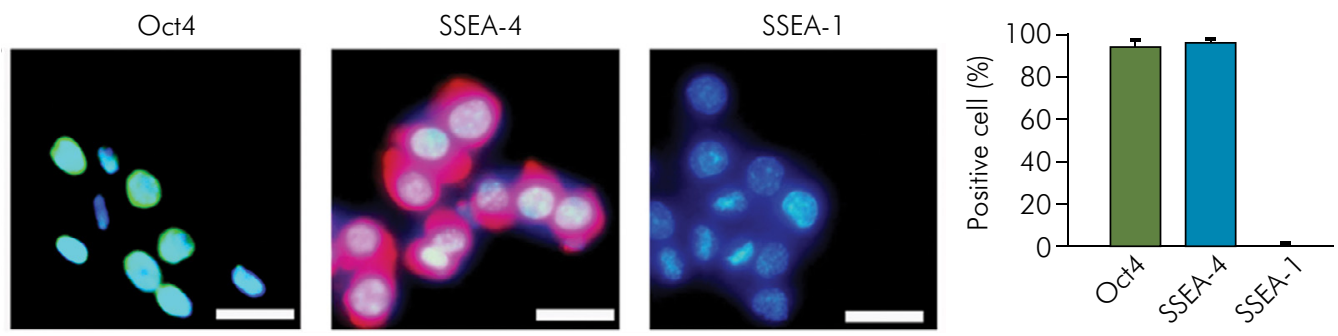

B
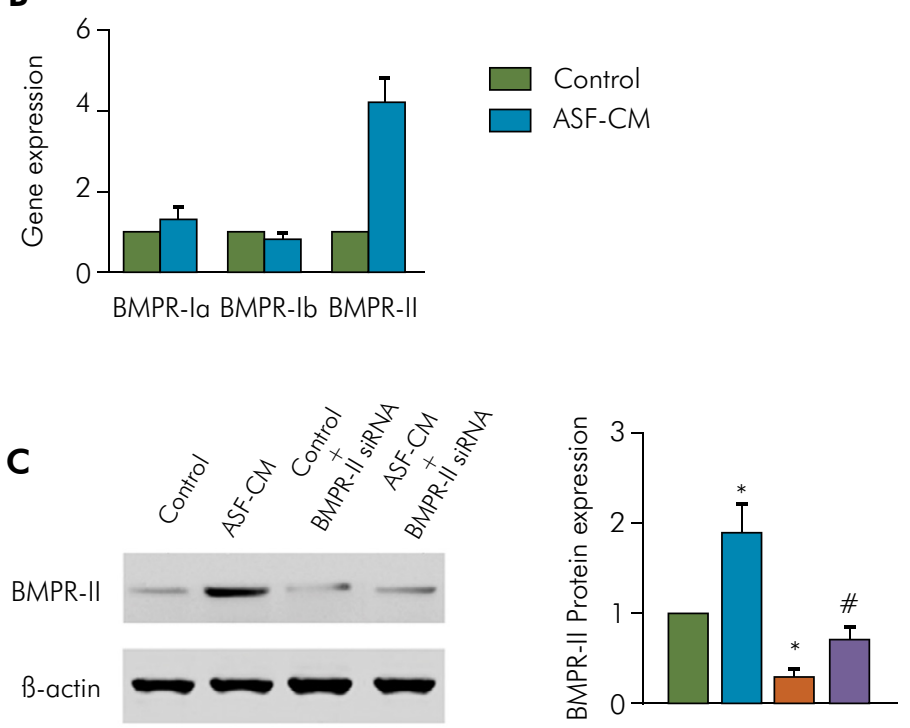

Control

ASF-CM

Control+BMPR-II siRNA

ASF-CM+BMPR-II siRNA

Figure 1. Characterization of mouse iPS cells and the gene and protein levels of BMP receptors in iPS cells induced by the epithelial cell medium (Control) and ameloblast-conditioned medium (ASF-CM). A: The immunofluorescent staining and cell counting of the expression of OCT4, SSEA-4 and SSEA-1 by iPS cells. B: RT-PCR analysis of the gene expression of BMP receptors in iPS cells induced by the control medium and ASF-CM. C: Western blotting analysis and quantification of the protein levels of BMPR-II in iPS cells induced by the control medium, ASF-CM, control medium+BMPR-II siRNA, or ASF-CM+BMPR-II siRNA. Scale bar represents $50 \mu \mathrm{m}$. ${ }^{*} p<0.05$, vs. control group. ${ }^{*} P<0.05$, vs. ASF-CM group. Characterization of the ameloblasts used to prepare serum-free conditioned medium (ASF-CM). Immunofluorescent staining showed that almost all cells are positive for the ameloblastic makers (ameloblastin and enamelin). Scale bar represents $25 \mu \mathrm{m}$. 
- Smad1/5 signal transduction regulates the ameloblastic differentiation of induced pluripotent stem cells

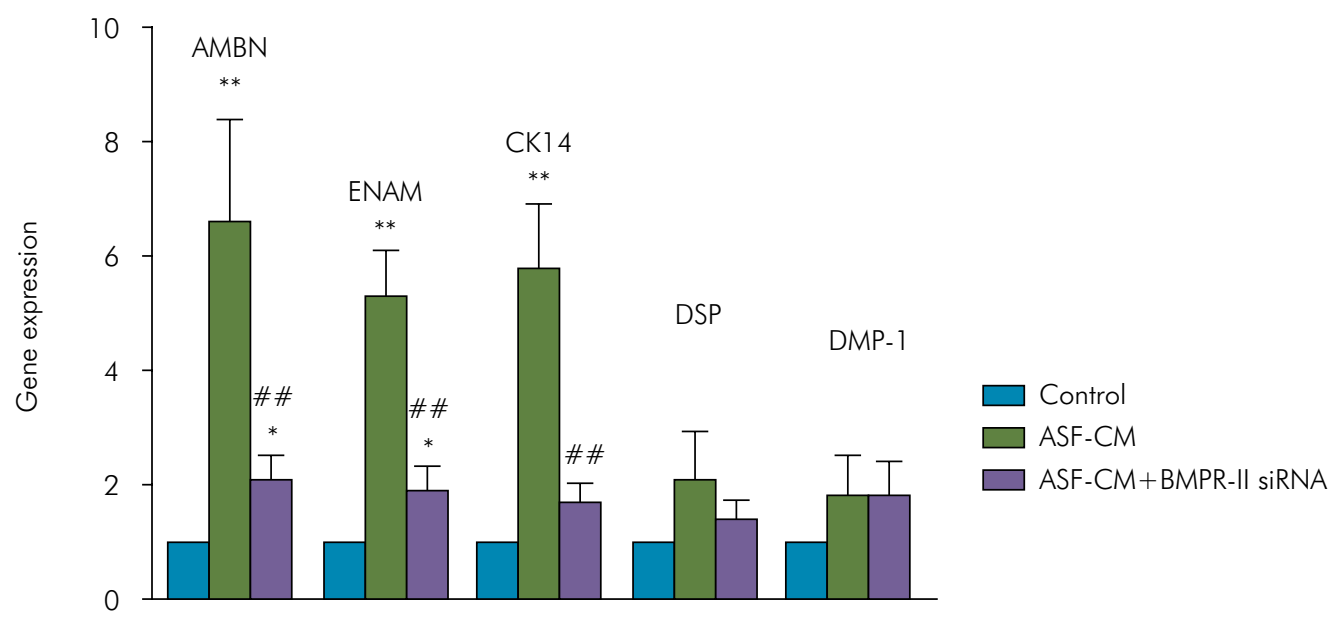

${ }^{*} p<0.05$, vs. control group. ${ }^{* *} p<0.01$, vs. control group. ${ }^{\#} p<0.01$, vs. ASF-CM group.

Figure 2. RT-PCR analysis of the expression of ameloblast-specific genes (Ambn, ENAM, and Ck 14) and odontoblast-specific genes (Dmp- 1 and Dsp) in iPS cells induced by the control medium, ASF-CM, or ASF-CM+BMPR-II siRNA.

A

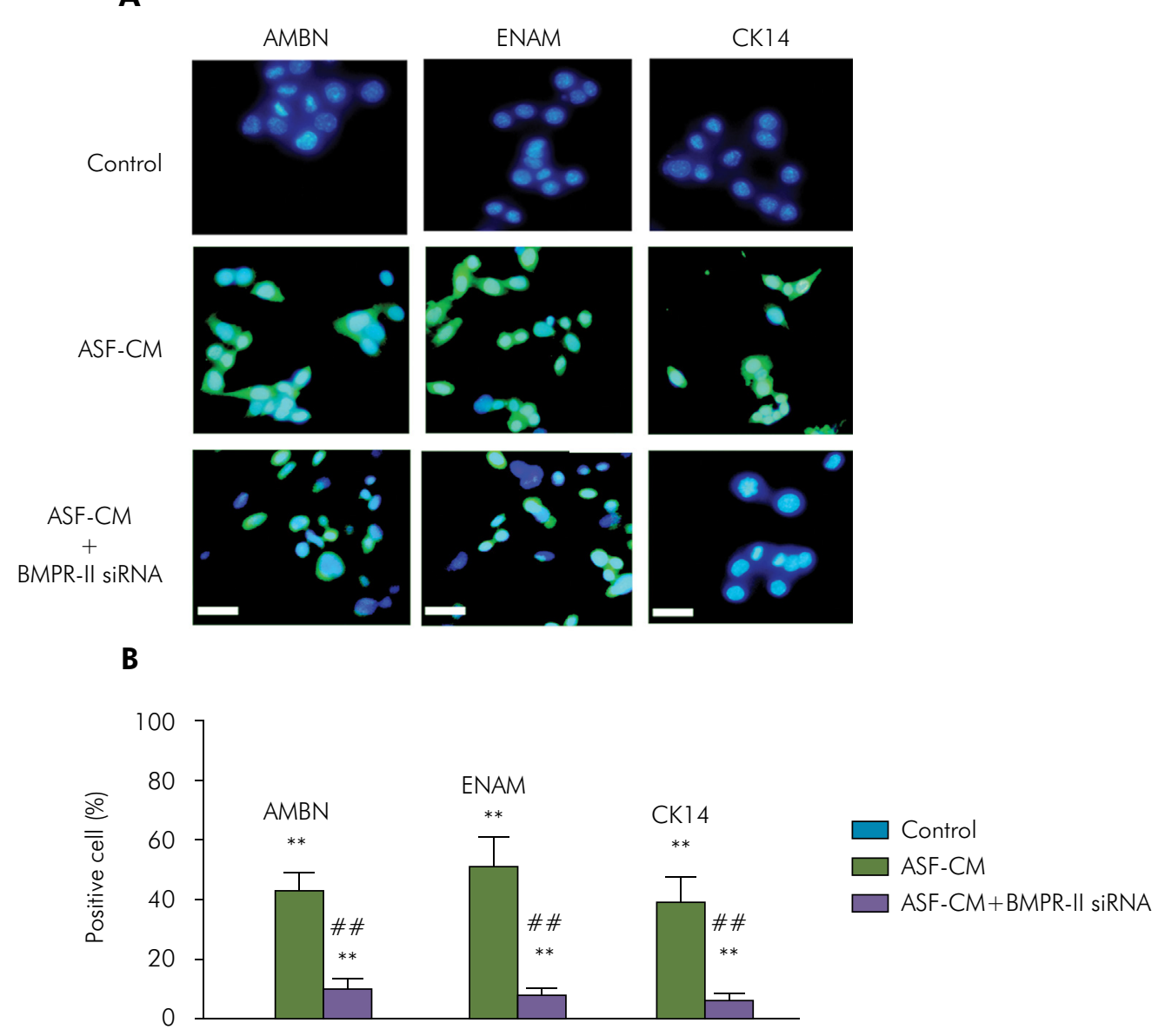

${ }^{*} p<0.01$, vs. control group. ${ }^{\# \#} p<0.01$, vs. ASF-CM group.

Figure 3. Immunofluorescent staining and quantification of the expression of AMBN, ENAM, and CK14 in iPS cells induced by the control medium, ASF-CM, or ASF-CM+BMPR-II siRNA. Scale bar represents $50 \mu \mathrm{m}$. 

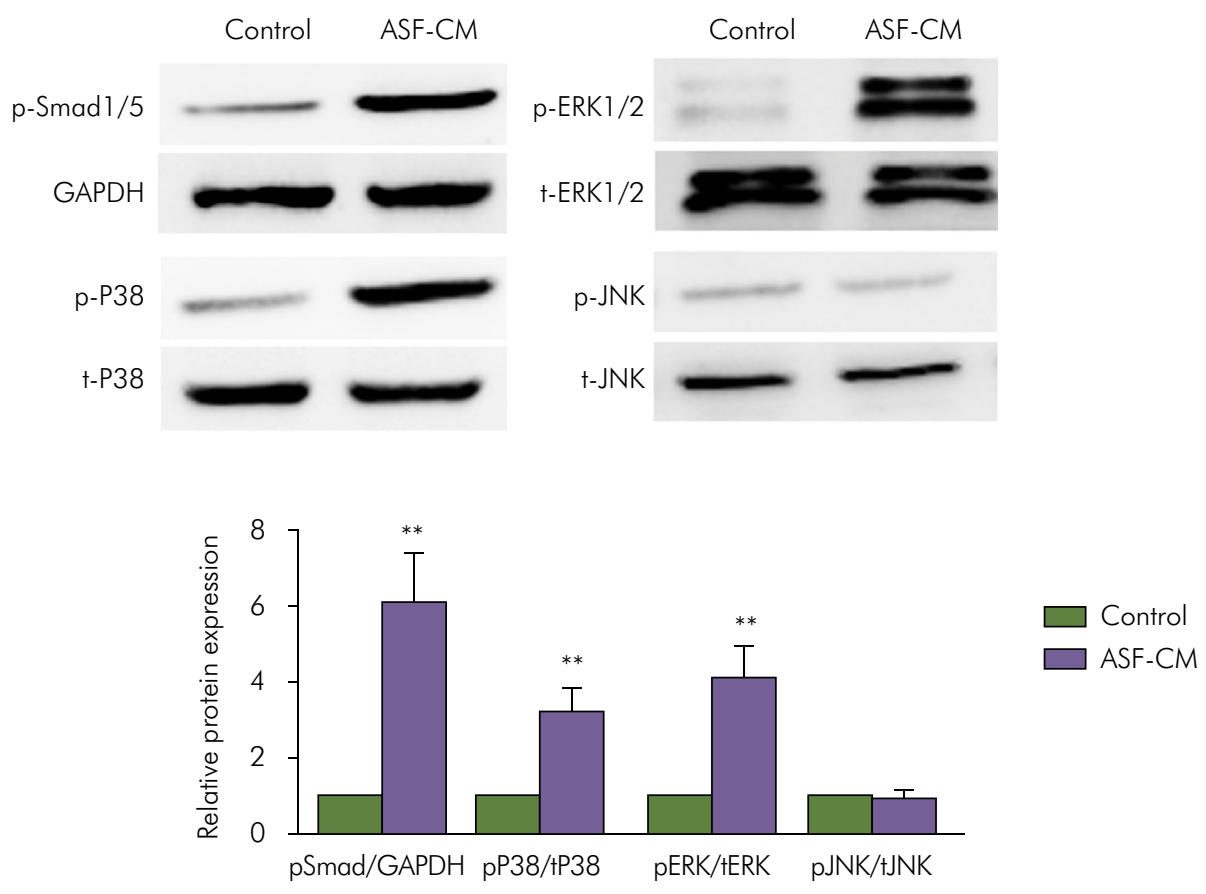

Figure 4. Western blotting analysis and quantification of the phosphorylation level of SMAD1/5 and P38, ERK1/2, and JNK MAPKs pathways in iPS cells induced by control medium or ASF-CM. ${ }^{* *} p<0.01$, vs. control group.

and ASF-CM+BMPR-II siRNA groups ( $\mathrm{p}>0.05$, Figure 2). Immunofluorescent staining showed that the iPS cells cultured in control medium were almost completely negative for AMBN, ENAM, and CK14 expression, while those cultured in ASF-CM showed strong cytoplasmic staining for AMBN, ENAM, and CK14 (Figure 3). Interestingly, the ASF-CM induced expression of AMBN, ENAM, and CK14 was also attenuated by the BMPR-II siRNA (all $p<0.05$, Figure 3). However, the expressions of DMP-1 and DSP were not detected in the above three groups (data not shown). The quantitative data showed that ASF-CM stimulation increased the protein levels of AMBN, ENAM, and CK14 significantly compared with those in the control, while the BMPR-II siRNA attenuated the above ASF-CM-induced effects significantly (all $\mathrm{p}<0.05$, Figure 3).

\section{Ameloblast-conditioned medium activated SMAD1/5, P38, and ERK1/2 signal transduction during ameloblastic differentiation of iPS cells}

To investigate the molecular mechanisms during ameloblastic differentiation of iPS cells via
BMPR-II activation, the phosphorylation of SMAD1/5 and MAPKs pathways were detected. The result showed that ASF-CM stimulation increased the phosphorylation of SMAD1/5, P38, and ERK1/2 significantly compared with the control (all $\mathrm{p}<0.05$, Figure 4); however, the phosphorylation of p-JNK in the ASF-CM stimulation group and the control group were similar (all $\mathrm{p}>0.05$; Figure 4).

\section{SMAD1/5 phosphorylation, but not P38 or ERK1/2 phosphorylation, is involved in the ameloblastic differentiation of iPS cells induced by the ameloblast- conditioned medium}

To investigate whether the SMAD1/5 and/or the P38 or ERK1/2 pathways are involved in the ameloblastic differentiation of iPS induced by the ameloblast-conditioned medium, inhibitors of SMAD1/5, P38 or ERK1/2 phosphorylation were used to stimulate iPS cells in combination with ASF-CM treatment. The results showed that SMAD1/5 inhibitor LDN-193189 decreased substantially the gene and protein expression of AMBN, ENAM, and CK14 induced by ASF-CM (all 


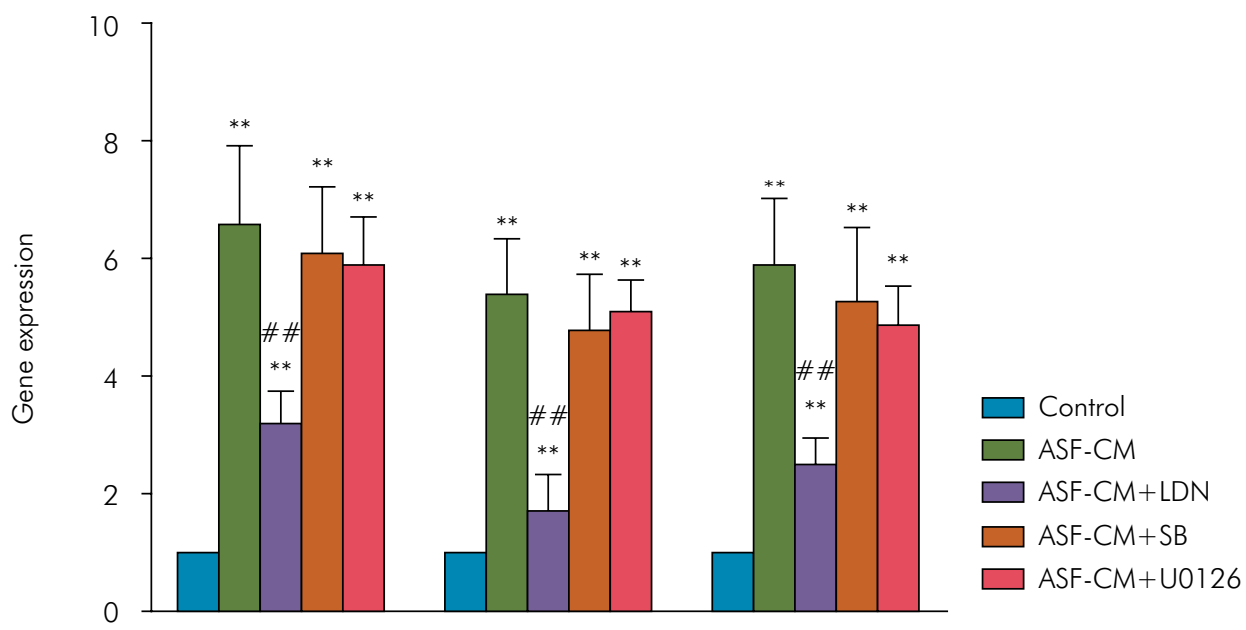

${ }^{* *} p<0.01$, vs. control group. ${ }^{\# \# p}<0.01$, vs. ASF-CM group.

Figure 5. RT-PCR analysis of the expression of Ambn, ENAM, and Ck14 of iPS cells induced by the control medium, ASF-CM, or ASF-CM respectively supplemented with 200 nM LDN-193189 (LDN, a selective SMAD1/5 inhibitor), $10 \mu M$ SB203580 (SB, a selective P38 inhibitor), or $10 \mu \mathrm{M} \cup 0126$ (a selective ERK1/2 inhibitor).

$\mathrm{p}<0.05$, Figures 5 and 6), while neither the P38 nor ERK1/2 inhibitors had any blocking effect on the ASF-CM-induced increase in the expressions of AMBN, ENAM and CK14 at the gene or protein level (all $p>0.05$, Figure 5 and 6).

\section{Discussion}

Recent study showed that BMP4 is an essential growth factor for the effective induction of ameloblastic differentiation of iPS cells. ${ }^{7}$ In the present study, we showed, for the first time, that inhibition of BMPR-II, one of the major receptors of BMP4, could block the ameloblastic differentiation of iPS cells induced by ameloblast-conditioned medium, which was characterized by decreased gene and protein expression of ameloblast markers AMBN, ENAM, and CK14. Furthermore, although the ameloblastconditioned medium could phosphorylate the SMAD1/5, P38, and ERK1/2 MAPKs during the ameloblastic differentiation of iPS cells, only inhibition of SMAD1/5 phosphorylation by the inhibitor of ALK receptors, could reverse the ameloblastic differentiation of iPS cells. Taken together, the results showed that the ameloblastic differentiation of iPS cells induced by ameloblast-conditioned medium is a SMAD1/5-dependent, but MAPKs-independent, transcriptional event.
The results showed that iPS cells stimulated by ameloblast-conditioned medium for 14 days exhibited higher gene and protein levels of ameloblast markers AMBN, ENAM, and CK14, but a very low level of odontoblast markers DMP-1 and DSP, which agreed with previous results.7 These results suggested that cultures using conditioned medium from ameloblasts could induce iPS cells to differentiate into cell types that closely resemble ameloblasts.

BMPR-II plays a key role in the BMP4 signaling pathway. ${ }^{13,14}$ Previous studies have suggested that the BMP4 signal regulates the formation of Hertwig's epithelial root sheath ${ }^{15}$ and the epithelial-mesenchymal interactions that occur during the patterning of the tooth crown and root. ${ }^{16}$ Meanwhile, BMPR-II is expressed substantially in the epithelial and mesenchymal cells of tooth germs at different stages of development. ${ }^{17-19}$ Therefore, in this study, we investigated the role of BMPR-II in ameloblastic differentiation of iPS cells induced by ameloblast-conditioned medium. As expected, the mRNA and protein levels of BMPR-II increased during the differentiation of iPS cells into ameloblasts. More importantly, silencing of BMPR-II using a specific siRNA reversed significantly the increased gene and protein levels of ameloblast markers AMBN, ENAM, and CK14 induced by the ameloblast-conditioned medium, confirming 

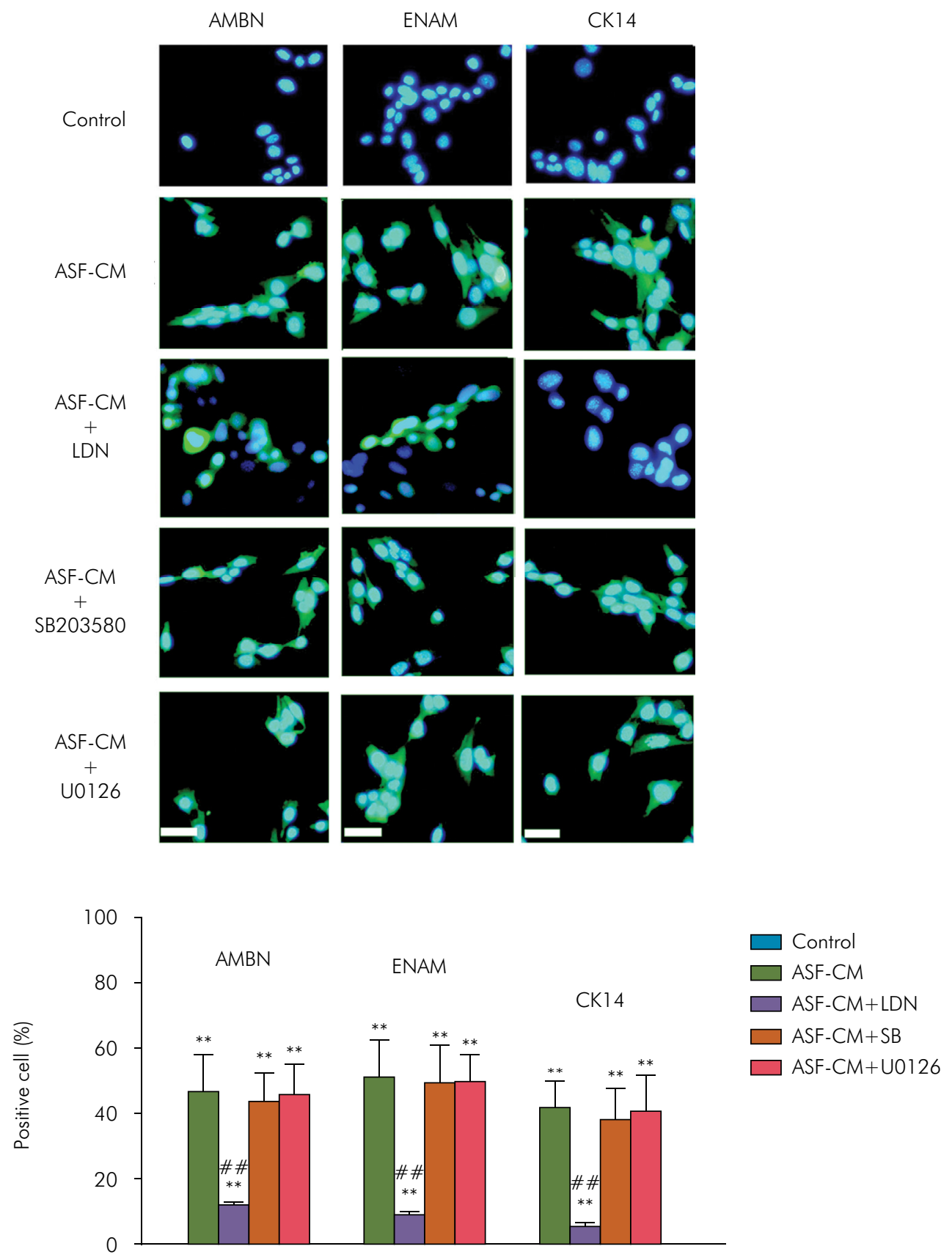

Figure 6. Immunofluorescent staining and quantification of the expression of AMBN, ENAM, and CK1 4 in iPS cells induced by the

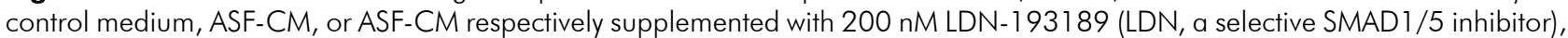
$10 \mu \mathrm{M}$ SB203580 (SB, a selective P38 inhibitor), or $10 \mu \mathrm{M}$ U0126 (a selective ERK1/2 inhibitor). Scale bar represents $50 \mu \mathrm{m}$. ${ }^{* *} \mathrm{P}<0.01$, vs. control group. ${ }^{\# \# P}<0.01$, vs. ASF-CM group.

that BMPR-II-mediated signal transduction plays a major role in the ameloblastic differentiation of iPS cells. Further study is needed to confirm the present phenomena by overexpressing BMPR-II transgene into iPS cells in the presence of ASF-CM. In addition, it was possible that factors present in
ASF-CM could increase the expression of BMPR-II through Alk-Smad1/5 signal transduction, and this molecular mechanism needs to be further clarified.

The intracellular signal transduction of BMPR-II has been studied extensively. Although activation of SMAD1/5 is regarded as the canonical BMP 
signal transduction, many physiological functions of BMP-II are achieved by MAPKs, including P38, ERK1/2, and JNK.,20 Therefore, the phosphorylation levels of SMAD1/5, and P38, ERK1/2, and JNK MAPKs were quantified to explore the mechanism by which BMPR-II regulates the ameloblastic differentiation of iPS cells. The western blotting results showed a significant increase in SMAD1/5, P38, and ERK1/2 phosphorylation after induction by ameloblast-conditioned medium. However, further analysis showed that only blockage of the SMAD1/5 pathway with the inhibitor of Alk receptors could decrease significantly the gene and protein levels of AMBN, ENAM, and CK14. Neither the P38 pathway inhibitor SB203580 nor the ERK1/2 inhibitor U0126 has any reversing effect on the ameloblastic differentiation of iPS cells. Therefore, collectively, our data indicated that the ameloblastic differentiation of iPS cells induced by ameloblast-conditioned medium was regulated primarily by SMAD1/5 signal pathways activated by Alk receptors. However, this fact does not reduce the participation of BMPR-II in the amaloblast differentiation process because it has been reported that there is dimerization effect between Alk and BmpRII receptors. ${ }^{20}$

The development and formation of teeth is very complicated and is regulated by many growth factors and signal pathways, and this dynamic process is unlikely to be manipulated only by the molecular factors identified to date. ${ }^{21-23}$ Therefore, in the present study, ameloblast-conditioned medium was used to induce ameloblastic differentiation of iPS cells. In addition, it has been reported that the P38 MAPK pathway is required for the effects of BMP2 and BMP7 on tooth development, such as the expression of p21 in the embryonic enamel knot and the expression of enamelin, ameloblastin, and $\beta 4$-integrin in ameloblasts; mice with a deletion of P38 in the ectoderm display defective secretion of dental enamel and the absence of dental cusps. ${ }^{24}$ ERK $1 / 2$ MAPK has been reported to play an important role in TGF- $\beta 1$-induced growth, collagen turnover, and differentiation of stem cells from tooth apical papilla. ${ }^{25}$ Therefore, further study is needed to identify the involvement of the P38 and ERK1/2 pathways in the regulation of ameloblastic differentiation of iPS cells induced by growth factors within the ameloblast-conditioned medium, such as BMP2, BMP7, and TGF- $\beta 1$.

There are some limitations in the present study. Firstly, only one murine iPS cell line was used, so the present results need to be confirmed by other iPS cell models. Secondly, although it has been shown that the ameloblast-conditioned medium contains several growth factors including BMPs, TGF- $\beta$ s, Notch-1, and fibroblast growth factors, ${ }^{26-28}$ the present study did not clarify the active components within the ASF-CM. In order to assure the reproducibility of the experiments, we have mixed together all the supernatants during ameloblasts culture, and then used them as ASF-CM for the subsequent experiments. In this way, the active components in the conditioned media were thought to be the same among the experiments. Further ELISA assays are needed to provide more details of the active components within the ASF-CM used in the present study. Thirdly, it is important to confirm the results using stably transfected clones of siRNA. However, the screening of such clones might affect the biological behaviors of iPS cells, so in the present study we only used the transient transfection, and the western blot results showed that the protein levels of BMPR-II have been efficiently decreased after $24 \mathrm{~h}$ transient transfection of BMPR-II siRNA. Furthermore, the transient transfection of BMPR-II siRNA significantly reversed the increased expression of ameloblastin, enamelin, and cytokeratin-14 induced by ASF-CM.

In summary, we have demonstrated that the smad1/5 signaling transduction, activated by ALK receptors, regulates the ameloblastic differentiation of iPS cells induced by ameloblast-conditioned medium. Further in vivo experimental studies are required to confirm the effect of the SMAD1/5 signal transduction on the ameloblastic differentiation of iPS cells.

\section{Acknowledgments}

This work was supported by grants from the Natural Science Foundation of Shannxi province ( $\mathrm{N}^{\mathrm{o}}$.2015014). 
Liu A, Zeng G, Qin Q, Sun C, Tan L

\section{References}

1. Smith EE, Yelick PC. Progress in bioengineered whole tooth research: from bench to dental patient chair. Curr Oral Health Rep. 2016 Dec;3(4):302-8. https://doi.org/10.1007/s40496-016-0110-2

2. Yamada Y, Nakamura S, Ito K, Sugito T, Yoshimi R, Nagasaka T, et al. A feasibility of useful cell-based therapy by bone regeneration with deciduous tooth stem cells, dental pulp stem cells, or bone-marrow-derived mesenchymal stem cells for clinical study using tissue engineering technology. Tissue Eng Part A. 2010 Jun;16(6):1891-900. https://doi.org/10.1089/ten.tea.2009.0732

3. Volponi AA, Pang Y, Sharpe PT. Stem cell-based biological tooth repair and regeneration. Trends Cell Biol. 2010 Dec;20(12):715-22. https://doi.org/10.1016/i.tcb.2010.09.012

4. Liu P, Zhang Y, Chen S, Cai J, Pei D. Application of iPS cells in dental bioengineering and beyond. Stem Cell Rev. 2014 Oct;10(5):66370. https://doi.org/10.1007/s12015-014-9531-2

5. Arakaki M, Ishikawa M, Nakamura T, Iwamoto T, Yamada A, Fukumoto E, et al. Role of epithelial-stem cell interactions during dental cell differentiation. J Biol Chem. 2012 Mar;287(13):10590-601. https://doi.org/10.1074/jbc.M111.285874

6. Yoshida K, Sato J, Takai R, Uehara O, Kurashige Y, Nishimura M, et al. Differentiation of mouse iPS cells into ameloblast-like cells in cultures using medium conditioned by epithelial cell rests of Malassez and gelatin-coated dishes. Med Mol Morphol. 2015 Sep;48(3):138-45. https://doi.org/10.1007/s00795-014-0088-6

7. Liu L, Liu YF, Zhang J, Duan YZ, Jin Y. Ameloblasts serum-free conditioned medium: bone morphogenic protein 4 -induced odontogenic differentiation of mouse induced pluripotent stem cells. J Tissue Eng Regen Med. 2016 Jun;10(6):466-74. https://doi.org/10.1002/ term. 1742

8. Bragdon B, Moseychuk O, Saldanha S, King D, Julian J, Nohe A. Bone morphogenetic proteins: a critical review. Cell Signal. 2011 Apr;23(4):609-20. https://doi.org/10.1016/i.cellsig.2010.10.003

9. Chen D, Zhao M, Harris SE, Mi Z. Signal transduction and biological functions of bone morphogenetic proteins. Front Biosci. 2004 Jan;9(1-3):349-58. https://doi.org/10.2741/1090

10. Bennett JH, Hunt P, Thorogood P. Bone morphogenetic protein-2 and -4 expression during murine orofacial development. Arch Oral Biol. 1995 Sep;40(9):847-54. https://doi.org/10.1016/0003-9969(95)00047-S

11. Yang K, Tang XD, Guo W, Xu XL, Ren TT, Ren CM, et al. BMPR2-pSMAD1/5 signaling pathway regulates RUNX2 expression and impacts the progression of dedifferentiated chondrosarcoma. Am J Cancer Res. 2016 Jun;6(6):1302-16.

12. Jiao K, Zeng G, Niu LN, Yang HX, Ren GT, Xu XY, et al. Activation of $\alpha 2 A$-adrenergic signal transduction in chondrocytes promotes degenerative remodelling of temporomandibular joint. Sci Rep. 2016 Jul;6(1):30085. https://doi.org/10.1038/srep30085

13. Pardali E, Ten Dijke P. TGF $\beta$ signaling and cardiovascular diseases. Int J Biol Sci. 2012;8(2):195-213. https://doi.org/10.7150/ijbs.8.195

14. Helbing T, Rothweiler R, Ketterer E, Goetz L, Heinke J, Grundmann S, et al. BMP activity controlled by BMPER regulates the proinflammatory phenotype of endothelium. Blood. 2011 Nov;118(18):5040-9. https://doi.org/10.1182/blood-2011-03-339762

15. Hosoya A, Kim JY, Cho SW, Jung HS. BMP4 signaling regulates formation of Hertwig's epithelial root sheath during tooth root development. Cell Tissue Res. 2008 Sep;333(3):503-9. https://doi.org/10.1007/s00441-008-0655-z

16. Huang XF, Chai Y. TGF-ß signalling and tooth development. Chin J Dent Res. 2010;13(1):7-15.

17. Kumamoto H, Ooya K. Expression of bone morphogenetic proteins and their associated molecules in ameloblastomas and adenomatoid odontogenic tumors. Oral Dis. 2006 Mar;12(2):163-70. https://doi.org/10.1111/j.1601-0825.2005.01177.x

18. Nadiri A, Kuchler-Bopp S, Perrin-Schmitt F, Lesot H. Expression patterns of BMPRs in the developing mouse molar. Cell Tissue Res. 2006 Apr;324(1):33-40. https://doi.org/10.1007/s00441-005-0120-1

19. Toyono T, Nakashima M, Kuhara S, Akamine A. Expression of TGF-beta superfamily receptors in dental pulp. J Dent Res. 1997 Sep;76(9):1555-60. https://doi.org/10.1177/00220345970760090701

20. Schmierer B, Hill CS. TGFbeta-SMAD signal transduction: molecular specificity and functional flexibility. Nat Rev Mol Cell Biol. 2007 Dec;8(12):970-82. https://doi.org/10.1038/nrm2297

21. Duailibi MT, Duailibi SE, Young CS, Bartlett JD, Vacanti JP, Yelick PC. Bioengineered teeth from cultured rat tooth bud cells. J Dent Res. 2004 Jul;83(7):523-8. https://doi.org/10.1177/154405910408300703

22. Honda MJ, Tsuchiya S, Sumita Y, Sagara H, Ueda M. The sequential seeding of epithelial and mesenchymal cells for tissue-engineered tooth regeneration. Biomaterials. 2007 Feb;28(4):680-9. https://doi.org/10.1016/i.biomaterials.2006.09.039

23. Oshima M, Tsuji T. Functional tooth regenerative therapy: tooth tissue regeneration and whole-tooth replacement. Odontology. 2014 Jul;102(2):123-36. https://doi.org/10.1007/s10266-014-0168-z

24. Greenblatt MB, Kim JM, Oh H, Park KH, Choo MK, Sano Y, et al. p38 $\alpha$ MAPK is required for tooth morphogenesis and enamel secretion. J Biol Chem. 2015 Jan;290(1):284-95. https://doi.org/10.1074/jbc.M114.599274 
Smad1/5 signal transduction regulates the ameloblastic differentiation of induced pluripotent stem cells

25. Chang HH, Chang MC, Wu IH, Huang GF, Huang WL, Wang YL, et al. Role of ALK5/Smad2/3 and MEK1/ERK signaling in transforming growth factor beta 1-modulated growth, collagen turnover, and differentiation of stem cells from apical papilla of human tooth. J Endod. 2015 Aug;41(8):1272-80. https://doi.org/10.1016/i.joen.2015.03.022

26. Harada H, Kettunen P, Jung HS, Mustonen T, Wang YA, Thesleff I. Localization of putative stem cells in dental epithelium and their association with Notch and FGF signaling. J Cell Biol. 1999 Oct;147(1):105-20. https://doi.org/10.1083/jcb.147.1.105

27. Tummers M, Thesleff I. Root or crown: a developmental choice orchestrated by the differential regulation of the epithelial stem cell niche in the tooth of two rodent species. Development. 2003 Mar;130(6):1049-57. https://doi.org/10.1242/dev.00332

28. Ning F, Guo Y, Tang J, Zhou J, Zhang H, Lu W, et al. Differentiation of mouse embryonic stem cells into dental epithelial-like cells induced by ameloblasts serum-free conditioned medium. Biochem Biophys Res Commun. 2010 Apr;394(2):342-7. https://doi. org/10.1016/j.bbrc.2010.03.007 